\title{
Fatal Rituximab-Induced Nonspecific Interstitial Pneumonia: Case Report and Review of the Literature
}

\author{
Rino Sato, MD, Jeremy Molligan, MD, and Sameh Gaballa, MD
}

\section{INTRODUCTION}

Rituximab is a chimeric anti-CD20 monoclonal antibody that is used to treat some hematological malignancies such as B-cell lymphomas, and various autoimmune diseases including immune thrombocytopenic purpura, systemic lupus erythematous, rheumatoid arthritis, and autoimmune hemolytic anemia. The most common side effects include fever, chills, and rigors. Respiratory complications such as cough, bronchospasm, sinusitis, and rhinitis have also been reported in $30 \%$ of patients in clinical trials. ${ }^{1}$ Rituximab-induced lung injury is a very rare but potentially fatal complication. We report a case of fatal single agent rituximab-induced nonspecific interstitial pneumonia to increase awareness about this serious side effect and review the current literature.

\section{CASE PRESENTATION}

This case involves a 72-year-old female with stage IV marginal-zone lymphoma (diffuse nodal and splenic involvement) who required therapy due to transfusiondependent anemia and was initiated on single-agent rituximab (four weekly doses at a dose of $375 \mathrm{mg} / \mathrm{m}^{2}$ ). She tolerated the first two infusions well, but started to feel short of breath after her third infusion. By the time she arrived to the infusion center for her fourth dose, she was short of breath at rest and was found to be hypoxic to $68 \%$ on room air. Of note, the patient had COPD with a 30 pack-year smoking history, quit three years ago, but had no previous oxygen requirement. She was admitted to the hospital where she was started on broad-spectrum antibiotics and placed on five liters of oxygen delivered via nasal cannula. A chest CT scan with contrast showed extensive bilateral ground-glass opacities with interlobular septal thickening (Figure 1a). On the second day of admission, the patient became hypoxic to $70 \%$ on six liters of supplemental oxygen, but did not tolerate bilevel positive airway pressure so she was transferred to the medical intensive care unit. Methylprednisolone 125 mg every six hours was started intravenously for possible rituximab-induced lung reaction in the intensive care unit. A repeat $C T$ scan of the chest two weeks later revealed improvement in the bilateral airspace opacities (Figure 1b).
However, she clinically continued to deteriorate during this time and she eventually required intubation and mechanical ventilation. Bronchoscopy was performed which was negative for infection including any aerobic or anaerobic bacteria, acid-fast bacilli, fungus, or virus. The patient further decompensated with hypoxemic respiratory failure and expired on the third week of admission despite multiple rounds of resuscitation. The patient's family agreed to a limited autopsy of her lung which revealed a histologic pattern of nonspecific interstitial pneumonia with uniform thickening of the alveolar septa with a monotonous lymphoid infiltrate. There was spatial and temporal homogeneity seen (Figure 2).

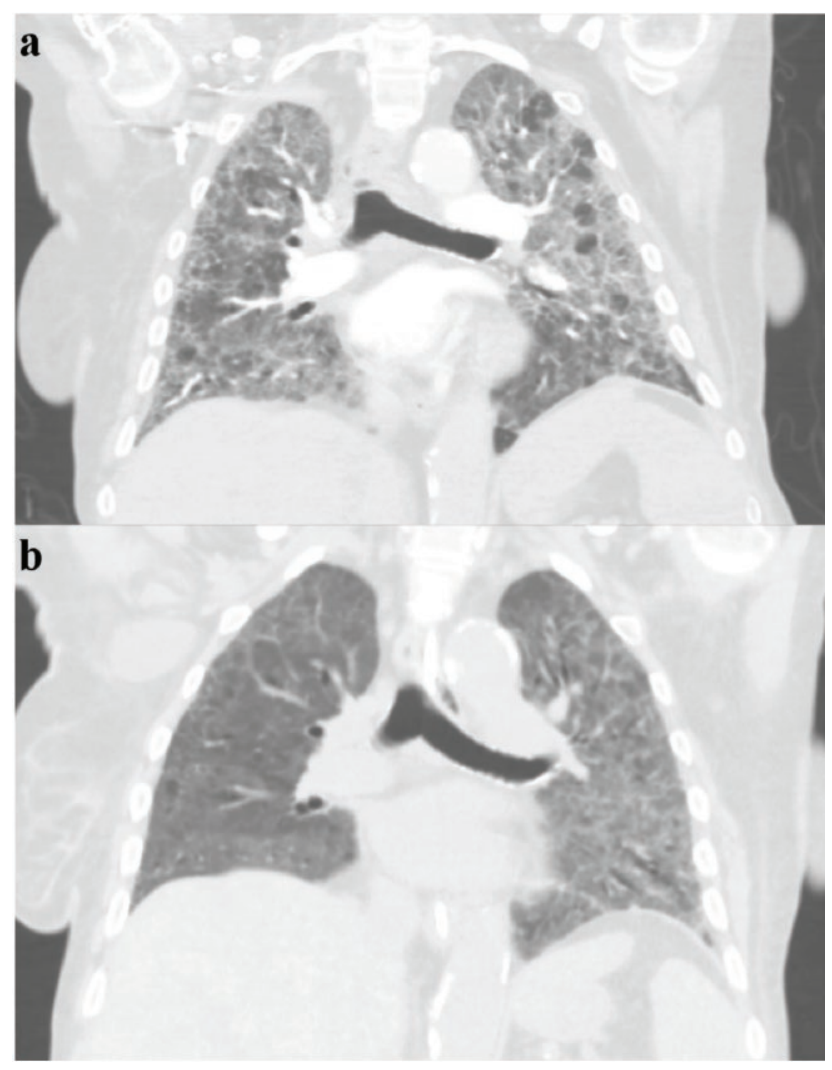

Figure 1. CT chest of this patient on admission (a) showed extensive ground glass opacification and septal thickening throughout the lungs bilaterally. (b) After two weeks of steroid therapy, the CT chest showed improvement in the extensive bilateral airspace opacities 


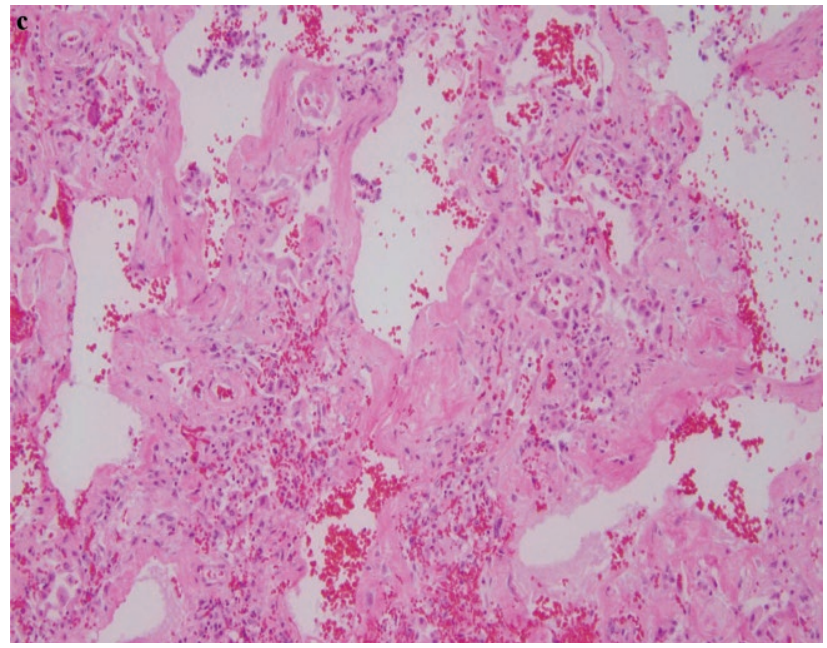

Figure 2. The biopsy of the autopsy lung showed histologic features of uniform interstitial inflammation with varying degrees of fibrosis consistent with nonspecific interstitial pneumonia.

\section{DISCUSSION}

Nonspecific interstitial pneumonia (NSIP) is a type of idiopathic interstitial pneumonia that is characterized by spatially and temporally uniform interstitial inflammation with varying degrees of fibrosis on pathology. ${ }^{6}$ NSIP can be idiopathic but can be associated with the toxic effects of drugs and occupational exposure. Rituximab has been reported in the literature to be a cause of NSIP, as well as other non-infectious-related interstitial lung diseases. . $^{2-5,7}$ The complicating factor with diagnosis and prognosis of rituximab-induced lung disease (RTX-ILD) is that rituximab is often used with concomitant chemotherapy or radiation which may also cause pulmonary toxicity, unlike our patient who only had rituximab monotherapy. Although several systematic reviews of the literature have noted RTX-ILD with rituximab monotherapy, only two reviews directly compared rituximab monotherapy to concomitant therapy. ${ }^{2,4,5,8}$ Blitzen et al. noted in a review of pediatric cases that the clinical presentations and outcomes were similar in patients with and without concomitant chemotherapy. ${ }^{7}$ Lioté et al. noted in their systematic review that nine patients experienced no further respiratory symptoms when chemotherapy was restarted without rituximab, implying that rituximab was the culprit for lung injury. ${ }^{5}$

The mechanism behind RTX-ILD is complex and remains unclear, but is thought to be secondary to complementdependent cell lysis, antibody dependent cellular toxicity, and tumor necrosis factor alpha release. 1,3,4,9,10,11 Rituximab efficiently eliminates B cells in lung tumors, normal lung tissues, and lung-associated lymph nodes, which may lead to normal lung injury through pro-inflammatory effects of activated cytokines, inflammatory mediators, and angiogenic factors. ${ }^{12,13}$ There may also be interference of lymphocyte crosstalk due to prolonged B-cell depletion, causing cytotoxic T lymphocyte dysregulation, thereby promoting lung damage. 2,14,15

Similar to our patient, typical RTX-ILD is subacute in onset becoming symptomatic after a median of 4.1 doses. ${ }^{2}$ The earliest presenting symptoms are often dry cough, exertional dyspnea, and fever. Common radiologic findings on CT scan include ground glass opacification, alveolitis, pulmonary fibrosis, alveolar hemorrhage, pleural effusion, and consolidation. Our patient did not get pulmonary function tests, but these would typically show a restrictive pattern on spirometry and significant DLCO reduction. Biopsy is not usually done but most commonly show pulmonary inflammation, and a range of histologic patterns can be seen including nonspecific interstitial pneumonia as was seen in our patient. ${ }^{1}$

The recommended treatment is prompt discontinuation of rituximab, initiation of steroids, and supportive therapy. However, there are no recommendations on dose, route, or duration of steroids. ${ }^{1-4,7,8}$ In a number of cases such as ours, high doses of steroids were unable to prevent death. ${ }^{2}$ Our patient received high-dose steroids on the second day of admission, but despite improvement on imaging after steroid initiation our patient ultimately expired. Improvement on her imaging suggests the steroids were treating the NSIP, but the treatment could have been initiated too late or the injury to an already diseased lung could have been too devastating.

If the patient survives an acute episode of RTX-ILD, it is unclear whether to avoid or reintroduce rituximab, as there have been cases of both positive and negative results. ${ }^{5}$ As for other treatment options, inhibiting the complement pathway or the activation of Nod-like receptor pyrin domain-containing protein 3 (NLRP3) inflammasome have also been discussed in the literature but no cases have been reported thus far. ${ }^{9,13}$ Wu et al. reported a case of RTX-ILD treated with methylprednisolone together with an antibody against tumor necrosis alpha therapy (Etanercept), but the benefits were only transient. ${ }^{3}$ Further investigation into treatment options and guidelines for RTX-ILD is necessary.

The risk factors for development of RTX-ILD remain unclear. Apart from Vulsteke et al. who reported a case of acute exacerbation of idiopathic pulmonary fibrosis with rituximab use, most studies have looked at RTX-ILD in patients with no previous lung injury. ${ }^{16}$ Franzen et al. also identified cigarette smoking as a possible risk factor for pulmonary function decline with rituximab and recommended smoking cessation during treatment. ${ }^{17}$ Our patient had an extensive smoking history and COPD and the further damage that rituximab had on her lung proved to be fatal despite intensive steroid therapy. Larger observational studies on the effect of rituximab on patients with prior pulmonary diseases or extensive smoking history are needed before recommending routine pre-treatment tests such as pulmonary function tests. 


\section{KEY POINTS}

Lung injury such as nonspecific interstitial pneumonia is a rare but serious side effect of rituximab. Awareness and education of providers and patients, close monitoring, and early detection and treatment are key to preventing fatal incidents of rituximab-induced lung injury (RTX-ILD). Routine assessment of lung function by pulmonary function tests should be performed during treatment with rituximab, especially for patients who have a previous history of lung disease. Suspicion for RTX-ILD should prompt discontinuation of the drug and a therapeutic trial of high-dose steroids, in order to prevent severe morbidity and mortality.

\section{REFERENCES}

1. Naqibullah M, Shaker SB, Bach KS, Bendstrup E. Rituximab-induced interstitial lung disease: five case reports. Eur Clin Respir J 2015;2.

2. Hadjinicolaou AV, Nisar MK, Parfrey H, Chilvers ER, Ostor AJ. Non-infectious pulmonary toxicity of rituximab: a systematic review. Rheumatology (Oxford) 2012:51:653-62.

3. Wu Y, Jia Y, Xu J, Shuai $X$, Wu Y. Fatal interstitial lung disease induced by rituximab-containing chemotherapy, treatment with TNF-alpha antagonist and cytokine profiling: a case-report and review of the literature. J Clin Pharm Ther 2013:38:249-53.

4. Wagner SA, Mehta AC, Laber DA. Rituximab-induced interstitial lung disease. Am J Hematol 2007:82:916-9

5. Liote H, Liote F, Seroussi B, Mayaud C, Cadranel J. Rituximab-induced lung disease: A systematic literature review. Eur Respir J 2010;35:681-7.

6. Kligerman SJ, Groshong S, Brown KK, Lynch DA. Nonspecific interstitial pneumonia: radiologic, clinical, and pathologic considerations. Radiographics 2009:29:73-87.
7. Bitzan M, Anselmo M, Carpineta L. Rituximab (B-cell depleting antibody) associated lung injury (RALI): a pediatric case and systematic review of the literature. Pediatr Pulmonol 2009:44:922-34.

8. Zayen A, Rais H, Rifi H, et al. Rituximab-induced interstitial lung disease: case report and literature review. Pharmacology 2011;87:318-20.

9. van der Kolk LE, Grillo-Lopez AJ, Baars JW, Hack CE, van Oers MH. Complement activation plays a key role in the side-effects of rituximab treatment. $\mathrm{Br} \mathrm{J}$ Haematol 2001;115:807-11.

10. Bienvenu J, Chvetzoff R, Salles G, et al. Tumor necrosis factor alpha release is a major biological event associated with rituximab treatment. Hematol J 2001:2:378-84

11. Smith MR. Rituximab (monoclonal anti-CD20 antibody): mechanisms of action and resistance. Oncogene 2003:22:7359-68.

12. Joly-Battaglini A, Hammarstrom C, Stankovic B, et al. Rituximab efficiently depletes B cells in lung tumors and normal lung tissue. F1000Res 2016;5:38.

13. Kong H, Wang Y, Zeng X, Zhu Q, Xie W, Dai S. Involvement of NLRP3 inflammasome in rituximab-induced interstitial lung disease: a case report. J Clin Pharm Ther 2014:39:691-4

14. Alexandrescu DT, Dutcher JP, O'Boyle K, Albulak M, Oiseth S, Wiernik PH. Fatal intra-alveolar hemorrhage after rituximab in a patient with non-Hodgkin lymphoma. Leuk Lymphoma 2004:45:2321-5

15. Selenko N, Maidic O, Draxier S, et al. CD20 antibody (C2B8)-induced apoptosis of lymphoma cells promotes phagocytosis by dendritic cells and cross-priming of CD8+ cytotoxic T cells. Leukemia 2001;15:1619-26.

16. Vulsteke C, Dierickx D, Verbeken E, Wolter P, Thomas J, Schoffski P. Rituximab-induced fatal interstitial pneumonitis: case report. Leuk Lymphoma 2010:51:546-8.

17. Franzen D, Ciurea A, Bratton DJ, et al. Effect of rituximab on pulmonary function in patients with rheumatoid arthritis. Pulm Pharmacol Ther 2016;37:24-9.

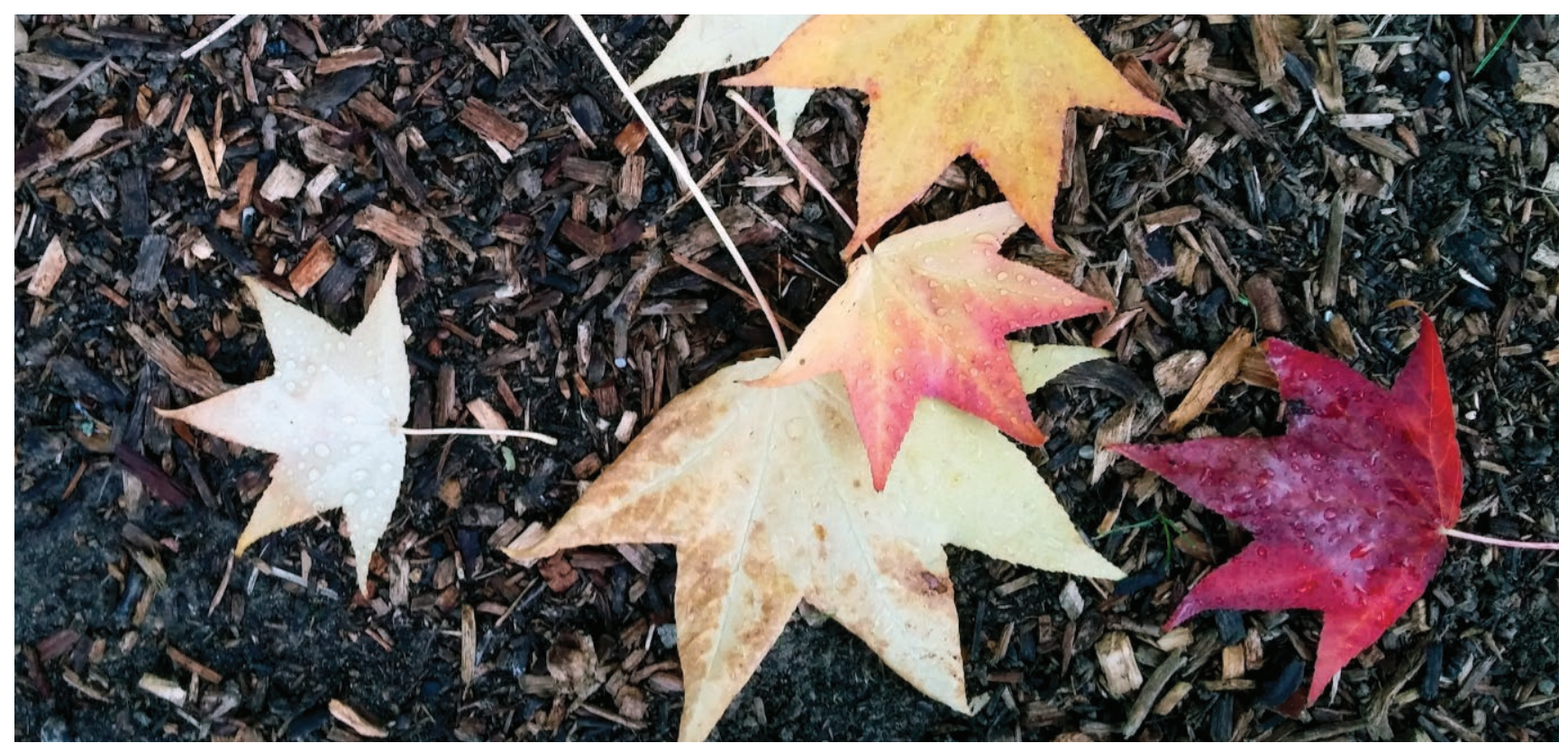

Anita Modi, MD 